\title{
Philosophiques
}

\section{Alan Thomas, Republic of Equals : Predistribution and Property-Owning Democracy, New York, NY, Oxford University Press, 2017, 472 pages}

\section{Gabriel Monette}

Volume 45, numéro 2, automne 2018

URI : https://id.erudit.org/iderudit/1055279ar

DOI : https://doi.org/10.7202/1055279ar

Aller au sommaire du numéro

Éditeur(s)

Société de philosophie du Québec

\section{ISSN}

0316-2923 (imprimé)

1492-1391 (numérique)

Découvrir la revue

Citer ce compte rendu

Monette, G. (2018). Compte rendu de [Alan Thomas, Republic of Equals : Predistribution and Property-Owning Democracy, New York, NY, Oxford University Press, 2017, 472 pages]. Philosophiques, 45(2), 527-532.

https://doi.org/10.7202/1055279ar d'utilisation que vous pouvez consulter en ligne.

https://apropos.erudit.org/fr/usagers/politique-dutilisation/ 


\title{
Comptes rendus
}

\author{
Alan Thomas, Republic of Equals: Predistribution and Property- \\ Owning Democracy, New York, NY, Oxford University Press, 2017, \\ 472 pages
}

Cet ouvrage ambitieux et érudit de la plume du philosophe britannique Alan Thomas est une contribution significative à la recherche sur le libéralisme, et plus généralement à la philosophie de l'économie ${ }^{1}$. Dans celui-ci, l'auteur offre non seulement une perspective originale sur l'œuvre du philosophe libéral John Rawls en y joignant plusieurs concepts issus de la conception républicaine de la liberté développée par Philip Pettit ${ }^{2}$, mais il y défend aussi une conception nouvelle provenant du mariage de ces deux approches: le libéralisme républicain.

La pertinence de cet ouvrage est d'autant plus grande qu'il dépasse le seul intérêt du mariage entre le libéralisme et le républicanisme, car il présente aussi une analyse rigoureuse des enjeux économiques de l'égalitarisme libéral rawlsien et du républicanisme. À cela se mêle une étude exhaustive des nombreux enjeux qui sont soulevés par l'application des principes de justice et les questions de domination dans la sphère économique. C'est surtout un travail impressionnant offrant de nombreux outils à celles et ceux qui voudraient interroger les rapports entre libéralisme et républicanisme. Thomas aborde en effet de manière détaillée de très nombreuses contributions aux questions liées à la liberté et au pouvoir dans la tradition tant libérale que républicaine. Les propositions d'égalitaristes aussi divers de Roemer, Ackerman ou Tomasi sont discutées en détail en offrant le cadre du libéralisme républicain comme base d'analyse critique. Ce livre offre donc en premier lieu une étude du libéralisme rawlsien appliqué aux questions économiques, qui s'inscrit dans un renouveau illustré par la récente publication de John Rawls: Reticent Socialist par William Edmundson ${ }^{3}$.

Il est important de noter que le républicanisme intervient régulièrement dans l'argumentaire de Thomas, mais toujours dans le rôle d'auxiliaire à l'argumentation, forme de réponse au danger de la domination et à la dérive oligarchique. En effet, chaque chapitre présente le libéralisme comme cadre d'analyse et invoque les idées républicaines comme support. Cela n'est

1. Alan Thomas, Republic of Equals: Predistribution and Property-Owning Democracy, New York, NY, Oxford University Press, 20I7 (Oxford political philosophy).

2. Philip Pettit, Républicanisme: une théorie de la liberté et du gouvernement, Paris, Gallimard, 2003 .

3. William A. Edmundson, John Rawls: Reticent Socialist, 2017.

PHILOSOPHIQUES 45/2 - Automne 2018, p. 527-574 
cependant pas le symptôme d'une méconnaissance des enjeux républicains. Sans brosser un portrait de toutes les approches républicaines, Thomas ne manque pas d'évoquer le républicanisme en entreprise (workplace republicanism) de Hsieh ou les perspectives critiques de McCormick. Il aurait pu être pertinent d'aborder cependant les travaux importants de Gourevitch ${ }^{4}$ sur le républicanisme du travail, ou les contributions de David Casassas et de Wispelaere ${ }^{5}$.

L'objectif du livre de Thomas est non seulement de développer cette approche qu'est le libéralisme, mais aussi de répondre à une question importante soulevée par Rawls dans Libéralisme politique 6 . Dans cet ouvrage, Rawls se pose la question suivante: quelle est la structure économique qui peut réaliser la justice comme équité ? Il conclut fameusement que, autant un système économique "socialiste de marché" qu'une "démocratie des propriétaires » peut réaliser la justice comme équité. Selon lui, l'un ou l'autre serait capable de réaliser ses idéaux. C'est cette conclusion que Thomas souhaite remettre en question. La thèse de l'auteur est claire. Il entend montrer que le socialisme de marché n'est, en fait, pas cohérent avec cette conception de la justice. Pour lui, la seule structure économique cohérente avec le libéralisme républicain est la démocratie des propriétaires. Autrement dit, Thomas considère, avec Rawls, que les concepts classiques du libéralisme ne nous permettent pas, à eux seuls, de choisir entre ces deux systèmes économiques. C'est seulement avec l'aide du républicanisme, de ses outils conceptuels et de sa sensibilité aux rapports de pouvoir, et avec l'aide de la notion de domination, qu'il est selon lui possible d'y arriver.

Il commence dans les trois premiers chapitres par une défense de Rawls en bonne et due forme. Ceux-ci présentent les concepts centraux qu'il espère tirer de Pettit et de Rawls, respectivement. On y voit alors que le travail de Thomas est positionné dans le camp rawlsien. Ces chapitres placent les bases de cette discussion, en abordant les questions de justice distributive posées par plusieurs commentateurs sur des questions comme l'optimum de Pareto, le rapport des individus avec la réalisation de la justice, etc.

C'est dans les deux chapitres suivants (IV et V) que Thomas ajoute les concepts républicains à la pensée rawlsienne. Dans cette partie du livre, Thomas critique la stratégie « isolationniste» de Cohen et de Rawls. Il considère exagérément optimiste l'idée que, pour protéger les libertés des individus, il est suffisant de les isoler du pouvoir généré par les inégalités permises par leurs théories. Il défend que le républicanisme apporte une stratégie plus efficace dans le monde réel en considération des tendances et des lois du

4. Alex Gourevitch, From Slavery to the Cooperative Commonwealth: Labor and Republican Liberty in the Nineteenth Century, Cambridge University Press, 2015.

5. David Casassas, et Jurgen de Wispelaere, «Republicanism and the Political Economy of Democracy ", European Journal of Social Theory, septembre 2015.

6. John Rawls, Libéralisme politique, Paris, PUF, 2016. 
monde social. Pour lui, il est nécessaire de structurer une société plus égalitaire dans la distribution des biens, notamment par une démocratie des propriétaires, pour diminuer la capacité effective des puissants à dominer et à s'attaquer aux libertés des individus.

Les quatre chapitres centraux (VI à X) portent sur l'économie du libéralisme républicain. L'auteur commence par une présentation de la démocratie des propriétaires et tente d'en offrir une interprétation égalitariste libérale et républicaine. Pour lui, la démocratie des propriétaires est l'idéal économique auquel tend le libéralisme républicain. Elle consiste en une structure économique dotant chaque individu d'une part de propriété lui servant à protéger ses libertés. Ce faisant, l'individu se dégage de la nécessité de se soumettre à l'arbitraire d'autrui. Il acquiert d'une certaine manière une plus grande autonomie au sens où il a un pouvoir de défection. C'est la contribution du républicanisme qui permet de choisir ce modèle plutôt que le socialisme de marché.

Le chapitre IX est central dans ce livre, car c'est le moment où l'argument de Thomas se déploie et où ce dernier apporte sa contribution à la théorie rawlsienne. C'est là qu'il développe sa critique du socialisme de marché et toute sa réflexion sur la question de la démocratie économique. Mais c'est aussi lors de la discussion du socialisme de marché que semble apparaître une faiblesse importante dans l'argumentaire de Thomas. En effet, la définition même qu'il donne de cette forme de socialisme est problématique, car il en choisit une radicale. Ce faisant, il peut clairement montrer comment son approche se distingue, mais ne peut pas sérieusement critiquer les autres formes plus modérées de socialisme de marché qui existent. En fait, son pluralisme ne semble pas clairement se distinguer d'approches socialistes comme celles décrites par le sociologue de l'économie Erik Olin Wright Alperovitz ${ }^{7}$.

Pour lui, le socialisme de marché est organisé de manière à ce que les seules institutions économiques soient des coopératives. Il ne manque pas d'évoquer d'autres propositions, mais celles-ci sont présentées comme moins «socialistes». Elles ne sont donc pas pour lui des candidates pour représenter le socialisme de marché. Son critère est donc particulièrement exigeant et semble manquer l'intuition rawlsienne qui consiste à penser que le socialisme de marché peut inclure d'autres mécanismes que les coopératives pour réaliser la justice, ou du moins à penser d'autres formes d'organisations économiques que celles qui existent actuellement.

Il importe de souligner que, tout au long de sa défense de la démocratie des propriétaires, Thomas ne manque pas de faire valoir les avantages d'une structure économique diversifiée tant en ce qui concerne les entreprises qu'en ce qui concerne les opportunités, mais il ne prend pas position sur la nécessité de favoriser une forme corporative par rapport à une autre.

7. Erik Olin Wright, Envisioning Real Utopias, London; New York, Verso, 20 Io. 
Pour lui, les institutions économiques, comme les entreprises qui émergeraient d'une démocratie des propriétaires libérale-républicaine ne pourraient fondamentalement pas dominer les individus. La capacité de sortie (exit), de "défection» des individus forcerait l'émergence d'entreprises plus soucieuses des droits de ceux-ci. Il n'est donc pas nécessaire d'avoir une conception plus forte de ce qu'est une entreprise plus juste, car la défection permet aux individus de se prémunir contre la domination qui pourrait avoir lieu au cœur des entreprises.

Or des commentateurs ${ }^{8}$ ont déjà montré qu'il n'était pas certain que la présence de la possibilité de défection, même significative, soit garante d'une protection contre toute forme de domination. De plus, nous avons des raisons de croire que la forme de l'entreprise est plus importante que la discussion de la défection peut le laisser croire. La manière dont le droit corporatif structure les entreprises n'est pas neutre. L'entreprise n'est pas simplement une institution servant à produire des biens et des services. Elle a une place particulière dans la société qui nécessite qu'on y réfléchisse comme étant une institution fondamentale de nos sociétés. Non seulement nous avons des raisons de croire que l'entreprise est une institution assez importante pour faire partie de la structure de base rawlsienne, même s'il y a encore des ambiguités liées à son statut ${ }^{9}$, mais une littérature se développe, surtout dans la perspective libérale, défendant l'idée que l'entreprise en tant qu'institution fondamentale à nos sociétés est un espace qui peut servir pour réaliser la justice ${ }^{10}$. C'est pourquoi il semble important de prendre au sérieux l'idée que la forme de l'entreprise a un rôle à jouer dans la réalisation de la justice et de la liberté républicaine. En fait, beaucoup de travail reste à faire en ce qui concerne le développement d'une forme d' "entreprise républicaine ", c'est-à-dire une entreprise structurée de manière à promouvoir la liberté républicaine.

Néanmoins, la critique que fait Thomas du socialisme de marché se base sur l'idée que les coopératives, seules institutions existant dans son interprétation du socialisme de marché, ouvrent la possibilité de rapports de domination entre les travailleurs. À ce titre, Thomas critique fermement les positions défendues par des auteurs comme Roemer ou Schweickart qui affirment qu'une société libre et juste serait exclusivement composée de coopératives. Tout en acceptant que ce soit une position radicale qui n'est pas représentative de la variété des propositions de socialisme de marché, Thomas montre que même dans les coopératives, type d'organisation réputée être plus juste, car plus près de ses travailleurs, il subsiste des formes

8. Nicholas Vrousalis, «Review of Thomas, Republic of Equals», 2017.

9. Sandrine Blanc, "L'entreprise fait-elle partie de la structure de base rawlsienne?", Revue de philosophie économique, vol. I 5, nº I, octobre 20I4, p. I67209.

10. Abraham Singer, The Form of the Firm: A Normative Political Theory of the Corporation, Oxford, New York, Oxford University Press, 2018. 
de domination. Or cette domination est à rejeter pour des raisons républicaines afin de favoriser plutôt une démocratie des petits propriétaires constituée de multiples mécanismes permettant aux individus propriétaires de bénéficier d'une certaine quantité de propriétés sous forme d'unités de «trust», de parts dans des entreprises publiques, etc. Ce modèle, que Thomas tire explicitement de Meade ${ }^{11}$, permettrait de donner aux individus les moyens de faire défection en cas de domination. Cela, doublé d'autres mécanismes d'inspiration républicaine, comme la contestation des décisions du management ${ }^{12}$, permettrait selon Thomas de réaliser les idéaux du libéralisme républicain. Le résultat, sur le plan institutionnel, de sa démocratie des propriétaires serait en fait une sorte de pluralisme, semblable à celui que propose Gal Alperovitz ${ }^{13}$.

Le chapitre $\mathrm{X}$ présente une critique sévère de l'approche libérale semblable à celle des libertariens que Tomasi développe dans Free Market Fair$n e s s^{14}$. Cette approche de la liberté économique n'a pour effet, selon Thomas, que de justifier la structure de l'économie que nous connaissons, comme si nous étions condamnés à vivre avec le niveau d'inégalités et de domination de la société actuelle et qu'il n'y avait que la guerre pour réellement atténuer la différence des conditions ${ }^{15}$.

Les derniers chapitres (XI à XII) abordent les questions plus pratiques de l'application d'une telle théorie. Le chapitre XI explore la question de l'utopisme. En effet, Thomas a, à travers le livre, défendu l'idée qu'il ne fallait pas se satisfaire de compromis intermédiaire, le second meilleur choix (second best) pour réaliser la justice, mais bien viser l'option idéale ${ }^{16}$. Le dernier chapitre examine la possibilité d'appliquer cette théorie dans le contexte contemporain marqué par de grandes inégalités et par la mondialisation. En effet, durant presque tout l'ouvrage, Thomas a développé une théorie inspirée de Rawls, c'est-à-dire tournée vers un État, et ce chapitre montre les limites de cette approche. Cependant, Thomas défend sa perspective en montrant que les approches localistes ou même municipalistes sont prometteuses et pourraient permettre de mettre en place des formes de démocratie des propriétaires.

11. James Meade, Liberty, Equality and Efficiency, London, Macmillan, I993; James Meade, Intelligent Radical's Guide to Economic Policy: the Mixed Economy, London, George Allen and Unwin, I975; James E. Meade, Efficiency, Equality and the Ownership of Property, Routledge, 20I2 (Routledge Revivals).

12. Nien-hê Hsieh, "Ralwsian Justice and Workplace Republicanism », Social Theory and Practice, janvier 2005.

13. Gar Alperovitz, et James Gustave Speth, America Beyond Capitalism: Reclaiming our Wealth, our Liberty, and our Democracy, Takoma Park, MA; Boston, MA, Democracy Collaborative Press, Dollars and Sense, 20I I.

14. John Tomasi, Free Market Fairness, 2013.

15. Thomas, Republic of Equals, p. 3 I4.

16. Ibid., p. 3 I6. 
Pour conclure, le livre peut être lu tant comme une défense de Rawls contre ses critiques, que comme une présentation de l'économie politique républicaine. C'est un projet ambitieux qui pose des questions importantes sur les institutions économiques de nos sociétés au regard de nos principes de justice. L'auteur déploie une connaissance encyclopédique de la littérature et des débats sur le libéralisme qui, à elle seule, mérite qu'on s'y intéresse. Outre la question de la forme des entreprises, qui fait défaut, peu de questions restent inexplorées. Quiconque veut se familiariser aux enjeux économiques du libéralisme ou du républicanisme est encouragé à parcourir ce livre.

GABRIEL MONETTE

Université de Montréal

Laure Cahen-Maurel, L'art de romantiser le monde. La peinture de Caspar David Friedrich et la philosophie romantique de Novalis, Zürich, LIT Verlag, 2017, 340 pages

L'ouvrage de Laure Cahen-Maurel, L'art de romantiser le monde, propose d'examiner le romantisme par-delà une certaine «vulgate» de celui-ci, à la lumière de deux figures a priori hétéroclites ${ }^{1}$ bien que contemporaines l'une de l'autre: celles du peintre Caspar David Friedrich (I774-I840) et du poète-philosophe Novalis (I772-I80I). Dans les premières pages du livre, Cahen-Maurel explique les motifs derrière le choix de ce double objet d'étude, non sans d'une part interroger les raisons pour lesquelles C. D. Friedrich est considéré aujourd'hui comme le représentant par excellence du romantisme en peinture, ni d'autre part sans justifier ce qui lui fait privilégier dans le cadre de sa réflexion la définition que donne Novalis du "romantisme ", plutôt que celle que l'on pourrait tirer des écrits des frères Schlegel (fondateurs de la revue l'Athenaeum et initiateurs du romantisme d'Iéna (I795-I797; I800-I 802), période particulièrement visée dans cette étude).

C'est en effet du fragment I05 des "Poéticismes» de Novalis que l'auteure tire un concept opératoire - en même temps outil herméneutique et fil conducteur - lui permettant de mener à la fois une exploration conceptuelle de l'imaginaire plastique du romantisme pictural de C. D. Friedrich, et une relecture double du romantisme par Novalis et de Novalis. L'auteure suggère ainsi la possibilité d'une réhabilitation philosophique du penseur dont l'impératif de romantisation du monde, tel que formulé au fragment I05, est à comprendre non pas comme simple élucubration poétique, mais comme résultant d'une intégration bien comprise de certains auteurs dont la pensée (surtout Kant et Fichte) précède la sienne. Il s'agit pour Cahen-

1. Laure Cahen-Maurel, L'art de romantiser le monde, Zürich, LIT Verlag, 20I7, p. 20. 